\title{
Stability of the Higgs sector in a flavor-inspired multi-scale model
}

\author{
Lukas Allwicher, Gino Isidori and Anders Eller Thomsen \\ Physik-Institut, Universität Zürich, \\ CH-8057 Zürich, Switzerland \\ E-mail: lukas.allwicher@physik.uzh.ch, isidori@physik.uzh.ch, \\ thomsen@itp.unibe.ch
}

ABSTRACT: We analyze the stability of the Higgs sector of a three-site model with flavornon-universal gauge interactions, whose spectrum of non-Standard-Model states spans three orders of magnitude. This model is inspired by deconstructing a five-dimensional theory where the generation index is in one-to-one relation to the position in the fifth dimension. It provides a good description of masses and mixing of the SM fermions in terms of scale hierarchies. We demonstrate that, within this construction, the mass term of the SM-like Higgs does not receive large corrections proportional to the highest mass scales. The model suffers only of the unavoidable "little hierarchy problem" between the electroweak scale and the lightest NP states, which are expected to be at the TeV scale.

Keywords: Beyond Standard Model, Higgs Physics, Quark Masses and SM Parameters

ARXIV EPRINT: 2011.01946 


\section{Contents}

1 Introduction 1

2 General considerations $\quad 3$

3 Simplified three-site model $\quad 3$

3.1 Higgs sector 4

3.2 Radiative stability of the Higgs-gauge sector 6

4 Realistic model $\quad 9$

$\begin{array}{lll}4.1 & \text { Flavor structure } & 10\end{array}$

$\begin{array}{lll}4.2 & \text { Stability } & 12\end{array}$

5 Conclusions 14

\section{Introduction}

Trying to stabilize the Standard Model (SM) Higgs sector against quantum corrections is a central theme in particle physics. The quadratic sensitivity of the Higgs mass term to ultraviolet degrees of freedom suggests the existence of new states around the $\mathrm{TeV}$ scale able to "screen" the SM ground state from possible higher scales. That we have not yet seen any new particle at the LHC experiments is posing a serious challenge to this general argument [1]. However, rather than completely abandoning the naturalness criterion [2] in this context, it is worth further investigating how serious the present challenge is.

A quantification of the tuning necessary to stabilize the Higgs mass in a given Beyond SM (BSM) framework is a model-dependent question that can be addressed only after specifying how heavy, and how strongly coupled to the SM fields, the new degrees of freedom are. Several analyses of this type exist in the literature for specific mechanisms focused on the stabilization of the Higgs sector (or, more precisely, the stabilization of the Higgs-gauge-top sector). In this paper we would like to address a different but closely related question, namely how addressing the flavor problem close to the electroweak scale affects the stability of the Higgs sector.

When considering the SM as a low-energy effective theory, we encounter a twofold issue in the flavor sector: there is no explanation for the hierarchical strucutre of the Yukawa couplings, and there are very severe bounds on possible non-SM sources of flavor violation [3]. We denote the combination of these two issues the flavor problem. In a wide class of BSM frameworks, the flavor problem and the stabilization of the Higgs sector are dealt with separately. The paradigmatic example is models based on the Minimal Flavor Violation (MFV) hypothesis [4,5]. Within this class of models, the new degrees 
of freedom stabilizing the Higgs sector are assumed to be flavor blind, but for correction terms proportional to the SM Yukawa couplings. While minimizing New Physics (NP) constraints from low-energy flavor-violating observables, nowadays the MFV hypothesis worsens the electroweak hierarchy problem, given that high- $p_{T}$ bounds are particularly strong for NP coupled in a family-universal way.

The opposite point of view is that of flavor-non-universal interactions already at the $\mathrm{TeV}$ scale. This approach is motivated by the smallness of light fermion masses: if we assume that the Yukawa interactions are the low-energy effect of some new dynamics, it is natural to consider a NP framework with a hierarchical spectrum with $\mathrm{TeV}$ scale dynamics coupled only to third generation fermions and heavier states coupled to the light SM fermions. A partial realization of this mechanism occurs in models with partial compositeness [6-9], see also [10]. However, in that context gauge interactions and, to a large extent, the dynamics stabilizing the Higgs sector, are still flavor universal. A more radical approach is that of flavor-non-universal gauge interactions, such as the $\mathrm{PS}^{3}$ framework proposed in [11]. The latter model, which is phenomenologically motivated by the recent $B$-physics anomalies (see e.g. [12] and references therein), is a prototype of the BSM framework we are interested in. On general grounds, this class of models has a clear advantage in escaping the NP bounds from direct searches. However, it might suffer from a more severe tuning in the Higgs sector due to the heavy states coupled to the light generations, which are charged under the SM gauge group. This potential problem is the issue we would like to investigate in this paper.

The underlying idea behind flavor-non-universal gauge interactions is that what we denote as "flavor" or "generation" is related to a specific localization in an extra space-like dimension. More precisely, following the principle of dimensional (de)construction [13], we can establish a one-to-one relation between the four-dimensional (4D) model and a five-dimensional (5D) theory where the fifth dimension is discretized, and the three flavor indices denote three notable points along this discretized dimension. For a more explicit construction of this type see e.g. ref. [14]. The 5D picture is instructive to provide a rational explanation for the peculiar structure of fields and couplings of the model, while the $4 \mathrm{D}$ description is more convenient in order to precisely compute amplitudes beyond the tree level.

In order to investigate the stability of the Higgs sector, in general terms, in this class of models, we employ the following strategy. In section 2 we briefly outline the rules on how to build renormalizable $4 \mathrm{D}$ models based on the idea of an underlying $5 \mathrm{D}$ theory, where flavor denotes the location in the fifth dimension. We then proceed checking explicitly how the scale mixes under quantum corrections in these $4 \mathrm{D}$ constructions. In section 3 we start analyzing a simplified model able to address the fermion mass hierarchies (relating them to different underlying scales), but not the fermion mixing. This allow us to introduce and discuss some general aspects of the construction and the stability of the Higgs-gauge sector. The issue of fermion mixing and the related stability of the Yukawa sector is discussed in section 4 within the context of a more realistic model. The results are summarized in the Conclusions. 


\section{General considerations}

The general structure of the models we are interested in is a chain of identical gauge groups, labeled by a flavor (or generation) index $i$, with each fermion family charged only under one of these gauge groups. This structure is inspired by the deconstruction in ordinary four-dimensional (4D) space time of models defined in $d=4+1$, where the fourth spacial dimension is compact and discretized into three lattice points corresponding to two fundamental intervals, or two fundamental scales. These points are in one-to-one correspondence with the flavor index. The gauge symmetry of the $5 \mathrm{D}$ theory reduces to a product of three identical 4D gauge groups, and a series of scalar (link) fields, $\Omega_{i(i+1)}$, transforming as bilinears of the (4D) gauge symmetry $[15,16]$. The vacuum expectation value (VEV) of the link fields breaks the chain of gauge groups to the diagonal (flavoruniversal) subgroup, which we identify with the electroweak SM gauge group, or at least with part of it.

To this underlying gauge and fermion structure, we add a series of Higgs fields, characterized by the flavor index $i$, which can be interpreted as the remnant of a unique 5D Higgs field with non-trivial dependence on the discretized dimension. We will not venture into the details of the 5D construction beyond the key point that tree-level interactions between different sites in the class of $4 \mathrm{D}$ models under consideration only occur via insertions of appropriate link fields. From the 5D perspective, this assumption can be viewed as the effect of fermion fields being localized on the three lattice points. The Higgs field responsible for the electroweak symmetry breaking (EWSB) is transmitted to the other sites through a slight mass mixing with the other Higgs states. Generically, this requires a hierarchical mass structure, which is reflected into an inverse hierarchy for the Higgs-Yukawa couplings thereby addressing the flavor problem. Similar constructions have also been pursued in fundamental $4 \mathrm{D}$ theories [17-19].

Our goal is to study the stability of the Higgs potential in the presence of such a mass hierarchy. For this reason, both in the simplified model analyzed in section 3 and in the more realistic model discussed in section 4 , we limit ourselves to consider a chain of $\mathrm{SU}(2)$ groups, with the diagonal $\mathrm{SU}(2)_{1+2+3}$ identified with the $\mathrm{SU}(2)_{\mathrm{L}}$ gauge group of the $\mathrm{SM}$. The rest of the SM gauge sector, which is not particularly relevant to our discussion, is treated as a flavor-universal group.

\section{Simplified three-site model}

The matter field content (fermions and scalars) of the simplified model is summarized in table 1. For simplicity, we consider only up-type quark singlets, though down-type can be added analogously. The Yukawa couplings for such fields, defined according to the rules specified above are

$$
\mathcal{L}_{Y}=-\sum_{i=1}^{3} y_{i} \bar{q}_{i, \mathrm{~L}} H_{i}^{*} u_{i, \mathrm{R}}+\text { H.c. }
$$

Assigning (flavor-universal) $\mathrm{U}(1)_{\mathrm{Y}}$ charges, as in the SM, to fermions and Higgs fields forbids any additional Yukawa interactions among the fields listed in table 1. The SM-like 


\begin{tabular}{|c|cc|}
\hline Fields & $\mathrm{SO}(1,3)$ & {$[\mathrm{SU}(2)]^{3}$} \\
\hline$q_{i}$ & $\left(\frac{1}{2}, 0\right)$ & $\mathbf{2}_{i}$ \\
$u_{i}$ & $\left(0, \frac{1}{2}\right)$ & $\mathbf{1}$ \\
\hline$H_{i}$ & 0 & $\mathbf{2}_{i}$ \\
$\Omega_{i(i+1)}$ & 0 & $\mathbf{2}_{i} \otimes \overline{\mathbf{2}}_{i+1}$ \\
\hline
\end{tabular}

Table 1. Matter field content of the minimal three-site model.

Higgs field, $\tilde{H}_{3}$, is the combination of the three $H_{i}$ fields that gets a non-vanishing VEV. Thanks to $\mathcal{L}_{Y}$ of eq. (3.1), this VEV gives rise to non-vanishing masses for all three fermion generations, whose values are controlled by $y_{i}$, assumed to be of $\mathcal{O}(1)$, and the Higgs mixing terms, which exhibit a hierarchical structure.

\subsection{Higgs sector}

The Higgs mixing is caused by the coupling of the $H_{i}$ 's to the link fields, $\Omega_{i j}$, whose VEVs break the gauge symmetry down to the diagonal subgroup. We will not go into the details of the link field potential but merely assume it to be such that they develop VEVs:

$$
\left\langle\Omega_{i(i+1)}\right\rangle=F_{i(i+1)}, \quad \text { with } \quad F_{12} \gg F_{23} \gg v_{\mathrm{EW}},
$$

where $v_{\mathrm{EW}}$ denotes the VEV of the SM-like Higgs. The relevant scalar couplings involving Higgs and link fields are

$$
\begin{aligned}
\mathcal{L} \supset & -\sum_{i=1}^{3} m_{i}^{2} H_{i}^{\dagger} H_{i}-\left(\sum_{i=1}^{2} \kappa_{i(i+1)} F_{i(i+1)} H_{i}^{\dagger} \Omega_{i(i+1)} H_{i+1}+\text { H.c. }\right) \\
& -\sum_{i=1}^{2} \lambda_{i}^{+} H_{i}^{\dagger} \Omega_{i(i+1)} \Omega_{i(i+1)}^{\dagger} H_{i}-\sum_{i=2}^{3} \lambda_{i}^{-} H_{i}^{\dagger} \Omega_{(i-1) i}^{\dagger} \Omega_{(i-1) i} H_{i}
\end{aligned}
$$

where $\kappa_{i(i+1)}$ and $\lambda_{i}^{ \pm}$are adimensional couplings. In a 5D description, all the interaction terms above can be derived starting from the 5D kinetic term of the unique Higgs field with non-trivial profile in the fifth dimension. However, the values of the couplings can be modified by adding non-trivial boundary conditions on the discrete points, which is why in the following we treat $\kappa_{i(i+1)}$ and $\lambda_{i}^{ \pm}$as free parameters. As we shall see, the consistency of the construction requires $\lambda_{2,3}^{-} \ll 1$, while all the other couplings can be of $\mathcal{O}(1)$.

From a pure $4 \mathrm{D}$ point of view, additional quartic couplings involving $\Omega_{i j}$ and $H_{i}$ are possible. However, our goal is to study the quadratic part of the effective Higgs potential and such terms do not contribute to it at the tree level, contrary to the terms in eq. (3.3), which do provide a contribution once the link fields acquire VEVs. ${ }^{1}$ To take into account

\footnotetext{
${ }^{1}$ From the underlying 5D construction, we expect quartic couplings involving only one type of Higgs field to be present at the tree level, whereas mixed terms of the type $\left(H_{i}^{\dagger} H_{i}^{\dagger}\right)\left(H_{j}^{\dagger} H_{j}^{\dagger}\right)$ to be radiatively generated and to appear in the potential with small couplings. In principle, the operator $H_{1}^{\dagger} \Omega_{12} \Omega_{23} H_{3}$ would modify the quadratic part of the Higgs potential already at the tree level; however, employing $5 \mathrm{D}$ arguments, we can assume the corresponding coupling to be very suppressed.
} 
the modification of the quadratic terms for $\left\langle\Omega_{i(i+1)}\right\rangle=F_{i(i+1)}$, it is convenient to redefine the $m_{i}^{2}$ and treat them as the effective tree-level Higgs masses after the link fields acquire VEVs. The stability of the potential under quantum corrections is discussed in the next subsection.

The key assumption on the various scales of the system (the link VEVs, $F_{i j}$, and the flavor-diagonal effective Higgs masses, $m_{i}$ ), which we employ in order to ensure both a correct hierarchy for the Yukawa couplings and a stable system under quantum corrections, is

$$
m_{1} \gg F_{12} \sim \varepsilon m_{1} \gg m_{2} \sim \varepsilon^{2} m_{1} \gg F_{23} \sim \varepsilon^{3} m_{1} \gg m_{3} \sim v_{\mathrm{EW}} \sim \varepsilon^{4} m_{1},
$$

where $\varepsilon$ is a small parameter of $\mathcal{O}\left(10^{-1}\right)$. As we shall see, $\varepsilon \sim(4 \pi)^{-1}$ is the smallest value for such parameter compatible with radiative stability. For $\lambda_{2,3}^{-}=\mathcal{O}(1)$, the scales in eq. (3.4) implies an $\mathcal{O}\left(\varepsilon^{2}\right)$ tuning between the $m_{i}^{2}$ before the link fields acquire VEVs, and the effective $m_{i}^{2}$ after the $[\mathrm{SU}(2)]^{3} \rightarrow \mathrm{SU}(2)_{1+2+3}$ symmetry breaking, for both $i=2$ and $i=3$. This is nothing but a manifestation of the little hierarchy problem [1] in this context. This tuning can be ameliorated assuming $\left|\lambda_{2,3}^{-}\right| \ll 1$, which in practice is equivalent to shifting the tuning to a different set of couplings. We will simply accept this tuning as a specific relation among couplings at low energies, as we address the question of stability under quantum corrections.

The hierarchy in eq. (3.4) allows us to perform a perturbative diagonalization of the $3 \times 3$ quadratic piece of the Higgs potential, namely

$$
\mu_{H}^{2}=\left(\begin{array}{ccc}
m_{1}^{2} & \kappa_{12} F_{12}^{2} & 0 \\
\kappa_{12} F_{12}^{2} & m_{2}^{2} & \kappa_{23} F_{23}^{2} \\
0 & \kappa_{23} F_{23}^{2} & m_{3}^{2}
\end{array}\right) \sim m_{1}^{2}\left(\begin{array}{ccc}
1 & \varepsilon^{2} & 0 \\
\varepsilon^{2} & \varepsilon^{4} & \varepsilon^{6} \\
0 & \varepsilon^{6} & \varepsilon^{8}
\end{array}\right)
$$

Defining

$$
\mu_{H}^{2}=O_{H} \operatorname{diag}\left(\tilde{\mu}_{1}^{2}, \tilde{\mu}_{2}^{2}, \tilde{\mu}_{3}^{2}\right) O_{H}^{\mathrm{T}} \equiv O_{H} \tilde{\mu}_{H}^{2} O_{H}^{\mathrm{T}}
$$

and keeping each entry to leading order (LO) in the $\varepsilon$ expansion, we obtain

$$
O_{H}=\left(\begin{array}{ccc}
1 & -\kappa_{12} \frac{F_{12}^{2}}{m_{1}^{2}} & \frac{\kappa_{12} \kappa_{23} F_{12}^{2} F_{23}^{2}}{m_{1}^{2} m_{2}^{2}-\kappa_{12}^{2} F_{12}^{4}} \\
\kappa_{12} \frac{F_{12}^{2}}{m_{1}^{2}} & 1 & -\frac{\kappa_{23} F_{23}^{2} m_{1}^{2}}{m_{1}^{2} m_{2}^{2}-\kappa_{12}^{2} F_{12}^{4}} \\
\frac{\kappa_{12} \kappa_{23} F_{12}^{2} F_{23}^{2}}{m_{1}^{4}} & \frac{\kappa_{23} F_{23}^{2} m_{1}^{2}}{m_{1}^{2} m_{2}^{2}-\kappa_{12}^{2} F_{12}^{4}} & 1
\end{array}\right) \sim\left(\begin{array}{ccc}
1 & \varepsilon^{2} & \varepsilon^{4} \\
\varepsilon^{2} & 1 & \varepsilon^{2} \\
\varepsilon^{8} & \varepsilon^{2} & 1
\end{array}\right),
$$

and

$$
\tilde{\mu}_{H}^{2}=\operatorname{diag}\left(m_{1}^{2}, m_{2}^{2}-\kappa_{12}^{2} \frac{F_{12}^{4}}{m_{1}^{2}}, m_{3}^{2}-\kappa_{23}^{2} \frac{F_{23}^{4} m_{1}^{2}}{m_{1}^{2} m_{2}^{2}-\kappa_{12}^{2} F_{12}^{4}}\right) \sim m_{1}^{2} \operatorname{diag}\left(1, \varepsilon^{4}, \varepsilon^{8}\right) .
$$

In order to trigger an SM-like EWSB, we require $\tilde{\mu}_{1,2}^{2}>0$ and $\tilde{\mu}_{3}^{2}<0$ with $\left|\tilde{\mu}_{3}\right|=\mathcal{O}(100 \mathrm{GeV})$. Since both terms in $\tilde{\mu}_{3}^{2}$ are of $\mathcal{O}\left(\varepsilon^{8}\right)$, this requirement does not imply any additional tuning. With a suitable choice of the parameters controlling quartic interactions, we can finally obtain $\left\langle\tilde{H}_{3}\right\rangle=v_{\mathrm{EW}}$ for the lightest Higgs field, which we identify with the SM-like Higgs boson. 
Returning to the flavor basis for the Higgs fields, it is easy to realize that the nonvanishing VEV of $\left\langle\tilde{H}_{3}\right\rangle$ is distributed to all the three fields in a hierarchical way. To leading non-vanishing order in the perturbative expansion, we get

$$
\left\langle H_{1}\right\rangle=\frac{\kappa_{12} \kappa_{23} F_{12}^{2} F_{23}^{2}}{m_{1}^{2} m_{2}^{2}-\kappa_{12}^{2} F_{12}^{4}}\left(\begin{array}{c}
0 \\
v_{\mathrm{EW}}
\end{array}\right), \quad\left\langle H_{2}\right\rangle=-\frac{\kappa_{23} F_{23}^{2} m_{1}^{2}}{m_{1}^{2} m_{2}^{2}-\kappa_{12}^{2} F_{12}^{4}}\left(\begin{array}{c}
0 \\
v_{\mathrm{EW}}
\end{array}\right), \quad\left\langle H_{3}\right\rangle=\left(\begin{array}{c}
0 \\
v_{\mathrm{EW}}
\end{array}\right),
$$

which, assuming $\mathcal{O}(1)$ values for the Yukawa couplings (3.1), leads to up-quark mass matrix

$$
m_{i j}^{u} \sim \operatorname{diag}\left(\varepsilon^{4}, \varepsilon^{2}, 1\right) v_{\mathrm{EW}} .
$$

The mechanism comfortably reproduces the observed hierarchy of the up-type quark masses with $\varepsilon \sim(4 \pi)^{-1}$.

The attentive reader will have noticed the absence of any mixing in the fermion mass matrix. The latter is not well defined in absence of $\mathrm{SU}(2)$-singlet down-type quarks, but it is easy to verify that adding such fields and proceeding in a similar manner does not lead to any physical mixing. This is because the fermion part of the Lagrangian respects a $\mathrm{U}(1)^{3}$ global symmetry of the fermions on each site protecting against any such mixing. The more realistic model introduced in section 4 will deal with this issue by introducing heavy vector-like fermions and, with them, fermion mixing.

\subsection{Radiative stability of the Higgs-gauge sector}

In order to discuss the stability of the Higgs potential under radiative corrections due to the gauge (and link) sector, we must first analyze the spectrum of the gauge fields in the model. The latter is generated by the kinetic terms of the link fields

$$
\mathcal{L} \supset \sum_{i=1}^{2} \operatorname{Tr}\left[D_{\mu} \Omega_{i(i+1)}^{\dagger} D^{\mu} \Omega_{i(i+1)}\right]
$$

which are responsible for the breaking $[\mathrm{SU}(2)]^{3} \rightarrow \mathrm{SU}(2)_{1+2+3}$. Defining $W_{i \mu}=W_{i \mu}^{a} \sigma^{a} / 2$, the covariant derivatives acting on the link fields are

$$
D_{\mu} \Omega_{i(i+1)}=\partial_{\mu} \Omega_{i(i+1)}-i g_{i} W_{i \mu} \Omega_{i(i+1)}+i g_{i+1} \Omega_{i(i+1)} W_{(i+1) \mu} .
$$

The mass terms for the gauge fields are then quickly determined by expanding the covariant derivatives in eq. (3.11):

$$
\mathcal{L} \supset \frac{1}{2} W_{i \mu}^{a}\left(M_{W}^{2}\right)_{i j} W_{j}^{a \mu}, \quad M_{W}^{2}=\left(\begin{array}{ccc}
g_{1}^{2} F_{12}^{2} & g_{1} g_{2} F_{12}^{2} & 0 \\
g_{1} g_{2} F_{12}^{2} & g_{2}^{2}\left(F_{12}^{2}+F_{23}^{2}\right) & g_{2} g_{3} F_{23}^{2} \\
0 & g_{2} g_{3} F_{23}^{2} & g_{3}^{2} F_{23}^{2}
\end{array}\right) .
$$

Diagonalization is performed first with an $\mathcal{O}(1)$ rotation between generations 1 and 2 , then with an $\mathcal{O}(1)$ rotation of 2 and 3 . After these, the remaining off-diagonal entries are removed with perturbative diagonalization. The result of this procedure is the rotation matrix

$$
O_{W}=\left(\begin{array}{ccc}
\frac{g_{1}}{g_{12}} & -\frac{g_{1} g_{2}^{2}}{g_{12} g_{123}^{2}} & \frac{g_{2} g_{3}}{g_{123}^{2}} \\
-\frac{g_{2}}{g_{12}} & -\frac{g_{1}^{2} g_{2}}{g_{12} g_{123}^{2}} & \frac{g_{3} g_{1}}{g_{123}^{2}} \\
\frac{g_{2}^{2} g_{3}}{g_{12}^{3}} \frac{F_{23}^{2}}{F_{12}^{2}} & \frac{g_{12} g_{3}}{g_{123}^{2}} & \frac{g_{1} g_{2}}{g_{123}^{2}}
\end{array}\right), \quad\left\{\begin{array}{l}
g_{12}^{2}=g_{1}^{2}+g_{2}^{2} \\
g_{123}^{4}=g_{1}^{2} g_{2}^{2}+g_{2}^{2} g_{3}^{2}+g_{3}^{2} g_{1}^{2}
\end{array}\right.
$$


where we have kept only the LO term in each entry. The resulting masses are

$$
\tilde{M}_{W}^{2}=O_{W}^{\mathrm{T}} M_{W}^{2} O_{W}=\operatorname{diag}\left(g_{12}^{2} F_{12}^{2}, g_{123}^{2} F_{23}^{2}, 0\right) \sim m_{1}^{2} \operatorname{diag}\left(\varepsilon^{2}, \varepsilon^{6}, 0\right) .
$$

We are now ready to analyze the stability of the mass term of the light Higgs field under radiative corrections generated by its interaction with gauge and link fields. Rather than analyzing the whole series of multi-loop corrections in a systematic way, it is convenient to start analyzing first the 2-3 sector in the limit of two sites only. We will work in the flavor basis, i.e. considering the fields $H_{2}$ and $H_{3}$, rather than the mass eigenstates $\tilde{H}_{2}$ and $\tilde{H}_{3}$, which differ from the former by a small mixing angle of $\mathcal{O}\left(\varepsilon^{2}\right)$, as shown in eq. (3.7).

We begin by considering the two-site limit before generalizing the discussion to the full model. After the diagonal symmetry breaking induced by the link fields, we are left with a massless gauge field, $\tilde{W}_{3 \mu}$, and a massive field, $\tilde{W}_{2 \mu}$, with mass $\tilde{M}_{W_{2}} \sim g F_{23} \sim \varepsilon m_{2}$. The 1-loop diagrams involving the heavy $\tilde{W}_{2}$ do not destabilize $m_{3}$, as can be understood by naïve dimensional considerations on the following topologies:

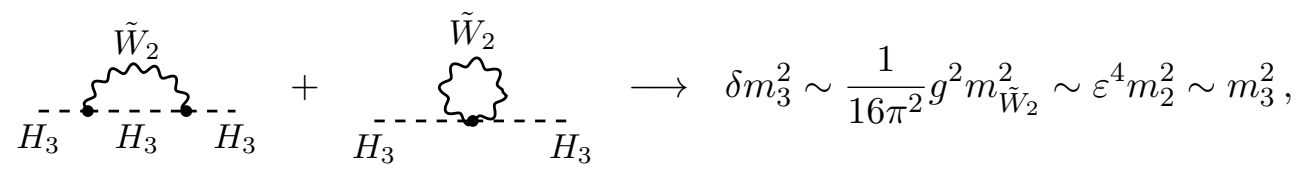

again with $\varepsilon=(4 \pi)^{-1}$. At the 2-loop order, we can consider diagrams of the type

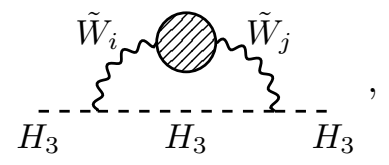

where

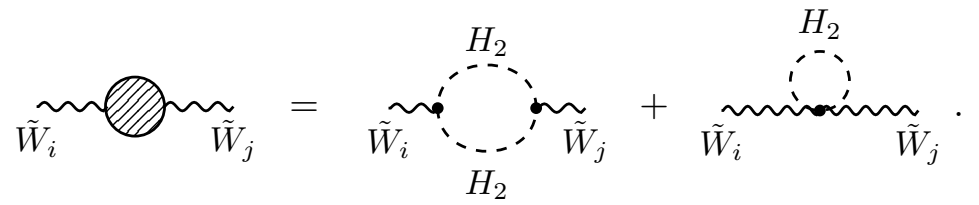

Although the 2-loop connection to the scale $m_{2}$ is already safe from our point of view (the two loops generate sufficient suppression), it is worth noticing that, $m_{2}$ being the heaviest mass in the diagram, we can in a first approximation ignore the vector boson masses in the propagators. One can then show that these leading terms cancel out completely when summing over all the possible gauge boson configurations. ${ }^{2}$ This is important when we consider the extension to three sites.

The trilinear coupling $\kappa_{23} F_{23} H_{2}^{\dagger} \Omega_{23} H_{3}$, being proportional to $F_{23}$, leads to a "soft" correction, not proportional to heavier scales:

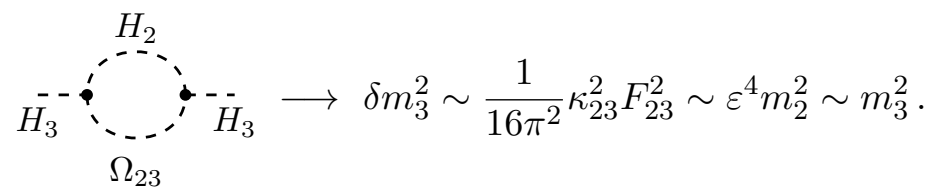

Note that the appearance of $m_{2}$ in (3.19) is purely conventional: the dimensionful coupling in the trilinear vertex implies only a logarithmic dependence from the heavy scale.

\footnotetext{
${ }^{2}$ This is readily seen in the gauge-basis for the vector bosons, where $W_{3}$ couples exclusively to $H_{3}$ and $W_{2}$ likewise to $\mathrm{H}_{2}$.
} 
A potential problem arises with

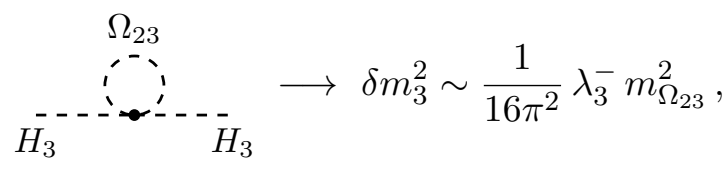

which might be dangerous depending on the value of $m_{\Omega_{23}}^{2}$, which we have not addressed yet. To this end we consider the leading 1-loop correction to the $\Omega_{23}$ mass

$$
\Omega_{\Omega_{23}} \stackrel{\tilde{W}_{2}}{\sum_{\Omega_{23}}} \longrightarrow \delta m_{\Omega_{23}}^{2} \sim \frac{1}{16 \pi^{2}} g^{4} F_{23}^{2} \sim m_{3}^{2} \text {, }
$$

from which we conclude that the two-site case is safe for what concerns corrections from the gauge-link sector.

We have now collected all the ingredients necessary to discuss the stability in the three-site case. Compared to the two-site case, the following generalizations can be made:

- In the three-site case there is a direct coupling of the light Higgs to the heavy $\tilde{W}_{1}$, i.e. the analog of the diagrams in (3.16) with $\tilde{W}_{2} \rightarrow \tilde{W}_{1}$. However, as we see from the gauge boson mixing matrix (3.14), this coupling is suppressed by $\left(F_{23}^{2} / F_{12}^{2}\right)^{2} \sim \varepsilon^{8}$, which is enough to render the contribution safe.

- Concerning the generalization of the diagram (3.17), we first observe that diagrams involving one or two $\tilde{W}_{1}$ are suppressed again by one or two factors of $\left(F_{23}^{2} / F_{12}^{2}\right)$. Moreover, we recall the argument about the cancellation of the leading contributions in the limit of vanishing gauge boson masses. The same reasoning also holds upon exchanging $H_{2}$ in the loop with $\Omega_{12}$ or $H_{1}$. These two arguments are sufficient to show that this class of diagrams does not spoil the hierarchy either.

- The 2-loop diagrams with scalars only connecting the scale $m_{3}$ with $m_{1}$, such as

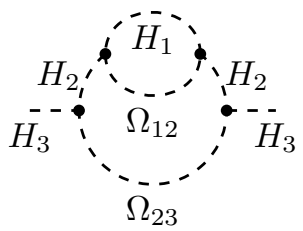

benefit from the suppression induced by the coupling, similarly to what happens for the diagram in (3.19). This makes them effectively 4-loop (from the point of view of the $\varepsilon$ counting), hence sufficiently suppressed contributions.

- The only diagram that becomes more problematic in the three-site case is the diagram (3.21), with $\tilde{W}_{2} \rightarrow \tilde{W}_{1}$, which results in a correction $\delta m_{\Omega_{23}}^{2} \sim m_{2}^{2}$. This affect the Higgs mass when nested into diagram (3.20) and would spoil the stability unless the following condition is satisfied:

$$
\text { I) } \quad \lambda_{3}^{-}=\mathcal{O}\left(\epsilon^{2}\right) .
$$

This condition is exactly the same condition we derived at the tree-level in order to ensure $m_{3} \sim v_{\mathrm{EW}} \ll F_{23}$. 


\section{Realistic model}

Having investigated the stability of the gauge-link sector in the simplified model, we are ready to extend the fermion sector of the model and consider an extended setup able to provide a realistic description of fermion mixing. For this purpose, we introduce a vector-like $\mathrm{SU}(2)$ doublet for each site, such that the matter content of the model is the content summarized in table 2. In terms of an extra-dimensional UV completion, these states can be identified with the ubiquitous Kaluza-Klein (KK) excitations associated with the quark doublets.

The fermionic part of the Lagrangian takes the form

$$
\begin{aligned}
\mathcal{L} \supset-\bar{Q} M Q-\left(\bar{Q}\left(\hat{y}_{\alpha}^{u} H_{\alpha}^{*}\right) u_{\mathrm{R}}+\bar{q}_{\mathrm{L}}\left(y_{\alpha}^{u} H_{\alpha}^{*}\right) u_{\mathrm{R}}+\bar{Q}\left(\hat{y}_{\alpha}^{d} H_{\alpha}\right) d_{\mathrm{R}}+\bar{q}_{\mathrm{L}}\left(y_{\alpha}^{d} H_{\alpha}\right) d_{\mathrm{R}}\right. \\
\left.+\bar{Q}_{L}\left(x_{a} \Omega_{a}\right) Q_{R}+\bar{q}_{L}\left(\hat{x}_{a} \Omega_{a}\right) Q_{R}+\text { H.c. }\right),
\end{aligned}
$$

where the fermion fields are to be understood as 3-component vectors in generation space, and $M=\operatorname{diag}\left(M_{1}, M_{2}, M_{3}\right)$. The $3 \times 3$ matrices of scalars and Yukawa couplings are given by (an identical expression holds for the $\hat{y}_{\alpha}^{u, d}$ )

$$
y_{\alpha}^{u, d} H_{\alpha}=\operatorname{diag}\left(y_{1}^{u, d} H_{1}, y_{2}^{u, d} H_{2}, y_{3}^{u, d} H_{3}\right) \text {, }
$$

and

$$
x_{a} \Omega_{a}=\left(\begin{array}{ccc}
0 & x_{12} \Omega_{12} & 0 \\
x_{12} \Omega_{12}^{\dagger} & 0 & x_{23} \Omega_{23} \\
0 & x_{23} \Omega_{23}^{\dagger} & 0
\end{array}\right), \quad \hat{x}_{a} \Omega_{a}=\left(\begin{array}{ccc}
0 & \hat{x}_{12} \Omega_{12} & 0 \\
\hat{x}_{21} \Omega_{12}^{\dagger} & 0 & \hat{x}_{23} \Omega_{23} \\
0 & \hat{x}_{32} \Omega_{23}^{\dagger} & 0
\end{array}\right) .
$$

We have let $\hat{x}_{i j} \neq \hat{x}_{j i}$ and $x_{i j}=x_{j i}$ to reflect the relations that holds for couplings originating from $5 \mathrm{D}$ profiles of the fermionic fields (for complex couplings we would have $x_{i j}=x_{j i}^{*}$ instead).

Mirroring the mass hierarchy of the scalar sector, the fermion mass terms are taken to be hierarchical with the heavy fields belonging to the first site. Here too, it is the role of the link fields to generate the off-diagonal mass terms

$$
M_{i j}=x_{i j} F_{i j} \quad \text { and } \quad \hat{M}_{i j}=\hat{x}_{i j} F_{i j}
$$

upon receiving VEVs. We find that a suitable ordering of the fermion mass terms that reproduce the flavor structure of the SM is given by

$$
M_{1} \sim m_{1} \gg M_{2} \sim \varepsilon m_{1} \gg M_{12}, \hat{M}_{12} \sim \varepsilon^{2} m_{1} \gg M_{3} \sim \varepsilon^{3} m_{1} \gg M_{23}, \hat{M}_{23} \sim \varepsilon^{4} m_{1},
$$

which can easily be accommodated setting the adimensional couplings $x_{i j}$ and $\hat{x}_{i j}$ of $\mathcal{O}(\varepsilon)$. This is perfectly consistent with the hierarchy of the scalar masses assumed in eq. (3.4). Note, however, that in the fermion sector we interchange the relative ordering between off-diagonal and diagonal mass terms: as we shall clarify below, this condition is necessary in order to avoid a too large mixing between the $Q_{\mathrm{L}}$ and $q_{\mathrm{L}}$ states. 


\begin{tabular}{|c|cc|}
\hline Fields & $\mathrm{SO}(1,3)$ & {$[\mathrm{SU}(2)]^{3}$} \\
\hline$q_{i}$ & $\left(\frac{1}{2}, 0\right)$ & $\mathbf{2}_{i}$ \\
$Q_{i}$ & $\left(\frac{1}{2}, 0\right) \oplus\left(0, \frac{1}{2}\right)$ & $\mathbf{2}_{i}$ \\
$u_{i}$ & $\left(0, \frac{1}{2}\right)$ & $\mathbf{1}$ \\
$d_{i}$ & $\left(0, \frac{1}{2}\right)$ & $\mathbf{1}$ \\
\hline$H_{i}$ & 0 & $\mathbf{2}_{i}$ \\
$\Omega_{i(i+1)}$ & 0 & $\mathbf{2}_{i} \otimes \overline{\mathbf{2}}_{i+1}$ \\
\hline
\end{tabular}

Table 2. Matter field content of the full model.

\subsection{Flavor structure}

With the introduction of the vector-like doublets $Q$, we open up for intergenerational mixing of the fermions via the link fields. We shall not endeavor to make a precision study of the resulting rotation matrices, as we are still working with a toy model. Rather, we wish to illustrate that the hierarchical texture of the Cabibbo-Kobayashi-Maskawa (CKM) matrix follows directly from the assumption of a hierarchy in the mass terms. As such, the texture can be expected to be a generic feature in all similar models.

We begin by determining the mass states of the left-handed quark doublets prior to EWSB. After the link fields acquire VEVs, $\left\langle\Omega_{a}\right\rangle=F_{a}$, the $6 \times 3$ mass term for the fermion doublets is of the form

$$
\mathcal{L} \supset-\left(\bar{q}_{\mathrm{L}} \bar{Q}_{\mathrm{L}}\right) M_{Q} Q_{\mathrm{R}}, \quad M_{Q}=\left(\begin{array}{c}
\hat{x}_{a} F_{a} \\
M+x_{a} F_{a}
\end{array}\right) .
$$

$M_{Q}$ has rank 3, ensuring three massless left-handed doublets, which are identified as SM fermions, while the massive states decouple from physics at the EW scale. The mass eigenstates of the fields are determined from the singular value decomposition of the mass matrix. Adopting the notation defined in (4.4), the rotation matrix $U_{\mathrm{L}}$, such that $U_{\mathrm{L}}^{\mathrm{T}} M_{Q} M_{Q}^{\mathrm{T}} U_{\mathrm{L}}$ is diagonal, is found to be ${ }^{3}$

$$
U_{\mathrm{L}}=\left(\begin{array}{cccccc}
1 & 2 \frac{\hat{M}_{12} \hat{M}_{23} M_{23}}{M_{2} M_{3}^{2}} & -2 \frac{\hat{M}_{12} \hat{M}_{32}}{M_{2}^{2}} & \frac{\hat{M}_{12} M_{12}}{M_{1}^{2}} & \frac{\hat{M}_{12}}{M_{2}} & -\frac{\hat{M}_{12} M_{23}}{M_{2} M_{3}} \\
-\frac{\hat{M}_{12} \hat{M}_{23} M_{23}}{M_{2} M_{3}^{2}} & 1 & 2 \frac{\hat{M}_{23} \hat{M}_{32} M_{23}}{M_{2} M_{3}^{2}} & \frac{\hat{M}_{21}}{M_{1}} & -\frac{\hat{M}_{21} M_{12}}{M_{1} M_{2}} & \frac{\hat{M}_{23}}{M_{3}} \\
\frac{\hat{M}_{12} \hat{M}_{32}}{M_{2}^{2}} & -\frac{\hat{M}_{23} \hat{M}_{32} M_{23}}{M_{2} M_{3}^{2}} & 1 & \frac{M_{12} \hat{M}_{32}}{M_{1}^{2}} & \frac{\hat{M}_{32}}{M_{2}} & -\frac{\hat{M}_{32} M_{23}}{M_{2} M_{3}} \\
\frac{\hat{M}_{12} M_{12}}{M_{1} M_{2}} & -\frac{\hat{M}_{21}}{M_{1}} & \frac{M_{12} \hat{M}_{32}}{M_{1} M_{2}} & 1 & -\frac{M_{12}}{M_{1}} & -\frac{\hat{M}_{21}}{M_{1} M_{33}} \\
-\frac{\hat{M}_{12}}{M_{2}} & \frac{\hat{M}_{21} M_{12}}{M_{1} M_{2}} & -\frac{\hat{M}_{32}}{M_{2}} & \frac{M_{12}}{M_{1}} & 1 & \frac{\hat{M}_{12}^{2} M_{23}}{M_{2}^{2} M_{3}}-\frac{M_{23}}{M_{2}} \\
\frac{\hat{M}_{12} M_{23}}{M_{2} M_{3}} & -\frac{\hat{M}_{23}}{M_{3}} & \frac{\hat{M}_{32} M_{23}}{M_{2} M_{3}} & \frac{M_{12} M_{23}}{M_{1}^{2}} & \frac{M_{23}}{M_{2}} & 1
\end{array}\right),
$$

\footnotetext{
${ }^{3}$ The degeneracy between the lightest massive and the three massless states is lifted at $\mathcal{O}\left(\varepsilon^{6}\right)$. The
} eigenvalues and leading eigenprojections, thus has to be determined to $\mathcal{O}\left(\varepsilon^{12}\right)$ to achieve $\mathcal{O}\left(\varepsilon^{6}\right)$ accuracy for $U_{\mathrm{L}}$. 
where, in order to keep the expression manageable, we show only the leading contribution in the $\varepsilon$ expansion for each entry. The left-handed doublet mass eigen-states are then given by

$$
\left(\begin{array}{c}
\tilde{q}_{\mathrm{L}} \\
\tilde{Q}_{\mathrm{L}}
\end{array}\right)=U_{\mathrm{L}}^{\mathrm{T}}\left(\begin{array}{c}
q_{\mathrm{L}} \\
Q_{\mathrm{L}}
\end{array}\right),
$$

and the parametric scaling of the different elements of $U_{\mathrm{L}}$ in powers of $\varepsilon$ is

$$
U_{\mathrm{L}} \sim\left(\begin{array}{cccccc}
1 & \varepsilon^{3} & \varepsilon^{4} & \varepsilon^{4} & \varepsilon & \varepsilon^{2} \\
\varepsilon^{3} & 1 & \varepsilon^{5} & \varepsilon^{2} & \varepsilon^{3} & \varepsilon \\
\varepsilon^{4} & \varepsilon^{5} & 1 & \varepsilon^{6} & \varepsilon^{3} & \varepsilon^{4} \\
\varepsilon^{3} & \varepsilon^{2} & \varepsilon^{5} & 1 & \varepsilon^{2} & \varepsilon^{3} \\
\varepsilon & \varepsilon^{3} & \varepsilon^{3} & \varepsilon^{2} & 1 & \varepsilon^{3} \\
\varepsilon^{2} & \varepsilon & \varepsilon^{4} & \varepsilon^{6} & \varepsilon^{3} & 1
\end{array}\right) .
$$

The mixing between the $q_{\mathrm{L}}$ and $Q_{\mathrm{L}}$ fields is responsible for populating the off-diagonal entries of the Yukawa coupling between the massless left-handed doublets and the righthanded singlets, $u_{\mathrm{R}}$ and $d_{\mathrm{R}}$. In terms of the mass eigenstates before EWSB, the Yukawa coupling for the up-type quarks reads

$$
\mathcal{L} \supset-\left(\begin{array}{ll}
\overline{\tilde{q}}_{\mathrm{L}} & \bar{Q}_{\mathrm{L}}
\end{array}\right) U_{\mathrm{L}}^{\mathrm{T}}\left(\begin{array}{c}
y_{\alpha}^{u} H_{\alpha}^{*} \\
\hat{y}_{\alpha}^{u} H_{\alpha}^{*}
\end{array}\right) u_{\mathrm{R}}
$$

At the EW scale the heavy $\tilde{Q}$ fields decouple and the resulting effecctive Yukawa coupling for the light (SM-like) fermions is contained in the upper $3 \times 3$ matrix. After EWSB, taking the hierarchy (3.9) of the Higgs VEVs, namely $v_{1} \sim \varepsilon^{4} v_{3}$ and $v_{2} \sim \varepsilon^{2} v_{3}$, and the structure (4.7) of $U_{\mathrm{L}}$ into account, leads to the mass matrix

$$
m_{u}=\left(\begin{array}{ccc}
y_{1}^{u} v_{1} & -\frac{\hat{M}_{12}}{M_{2}} \hat{y}_{2}^{u} v_{2} & \frac{\hat{M}_{12} M_{23}}{M_{2} M_{3}} \hat{y}_{3}^{u} v_{3} \\
-\frac{\hat{M}_{21}}{M_{1}} \hat{y}_{1}^{u} v_{1} & y_{2}^{u} v_{2} & -\frac{\hat{M}_{23}}{M_{3}} v_{3} \hat{y}_{3}^{u} \\
\mathcal{O}\left(\varepsilon^{7}\right) & -\frac{\hat{M}_{32}}{M_{2}} \hat{y}_{2}^{u} v_{2} & y_{3}^{u} v_{3}
\end{array}\right) \sim\left(\begin{array}{ccc}
\varepsilon^{4} & \varepsilon^{3} & \varepsilon^{2} \\
\varepsilon^{6} & \varepsilon^{2} & \varepsilon \\
\mathcal{O}\left(\varepsilon^{7}\right) & \varepsilon^{5} & 1
\end{array}\right) \times v_{\mathrm{EW}}
$$

of the up-type quarks. The rotation $V_{\mathrm{L}}^{u}$ required to bring the left-handed $u$ quarks to the mass basis can then be determined by singular value decomposition of $m_{u}$, or by the diagonalization of $m_{u} m_{u}^{\mathrm{T}}$. The latter can be done perturbatively in $\varepsilon$ : given the level of approximations employed so far, we can compute the eigenvalues of $m_{u} m_{u}^{\mathrm{T}}$ up to $\mathcal{O}\left(\varepsilon^{6}\right)$. Since the degeneracy of the two lightest squared masses is lifted only at order $\varepsilon^{4}$, this allows for determining $V_{\mathrm{L}}^{u}$ up to $\mathcal{O}\left(\varepsilon^{2}\right)$, which is the level of accuracy necessary for describing the structure of the CKM matrix.

The determination of $m_{d}$ and the left-handed rotation matrix $V_{\mathrm{L}}^{d}$ for the down quarks is entirely analogous to that in the up sector. In fact, since the structure of both $m_{u}$ and $m_{d}$ is determined by the $U_{\mathrm{L}}$ rotation, the mass matrix (4.11) is valid for $m_{d}$ upon substitution $y^{u}\left(\hat{y}^{u}\right) \rightarrow y^{d}\left(\hat{y}^{d}\right)$. One thing to bear in mind is that, within the SM, down-type quark masses exhibit a different hierarchy with respect to up-type ones. This feature can be 
implemented in the model with the minor adjustment $y_{2}^{d} \sim \hat{y}_{2}^{d} \sim y_{3}^{d} \sim \hat{y}_{3}^{d} \sim \varepsilon$. The result of this procedure is the physical CKM matrix

$$
V_{\mathrm{CKM}}=V_{L}^{u^{\dagger}} V_{L}^{d}=\left(\begin{array}{ccc}
1 & V_{12} & V_{13} \\
-V_{12} & 1 & V_{23} \\
V_{31} & -V_{23} & 1
\end{array}\right) \sim\left(\begin{array}{ccc}
1 & \varepsilon & \varepsilon^{2} \\
\varepsilon & 1 & \varepsilon \\
\varepsilon^{2} & \varepsilon & 1
\end{array}\right),
$$

where

$$
\begin{aligned}
V_{12} & =\frac{\hat{M}_{12}}{M_{2}} \Delta y_{2}, \quad \hat{2}_{23}=\frac{\hat{M}_{23}}{M_{3}} \Delta y_{3}, \\
V_{13} & =\frac{\hat{M}_{12}}{M_{2}}\left(\frac{\hat{M}_{23}}{M_{3}} \frac{\hat{y}_{2}^{u}}{y_{2}^{u}}-\frac{M_{23}}{M_{3}}\right) \Delta y_{3}, \\
V_{31} & =\frac{\hat{M}_{12}}{M_{2}}\left(\frac{M_{23}}{M_{3}}-\frac{\hat{M}_{23}}{M_{3}} \frac{\hat{y}_{2}^{d}}{y_{2}^{d}}\right) \Delta y_{3} .
\end{aligned}
$$

and

$$
\Delta y_{i}=\frac{\hat{y}_{i}^{u}}{y_{i}^{u}}-\frac{\hat{y}_{i}^{d}}{y_{i}^{d}}
$$

With $\varepsilon=(4 \pi)^{-1}$ and $\mathcal{O}(1)$ parameters in the range $\left[\frac{1}{3}, 3\right]$, the parametric structure in (4.12) fits well with the observed hierarchical structure of the CKM matrix [20]. Combining this fact with the compatibility of the eigenvalues of both $m_{u}$ and $m_{d}$ with observations, we conclude that this framework provides a realistic description of the SM quark Yukawa couplings. Moreover, the construction is such that both $m_{u}$ and $m_{d}$ are diagonalized by tiny right-handed rotations. To a large extent, the model provides an explicit dynamical realization of the minimally-broken $\mathrm{U}(2)^{3}$ quark-flavor symmetry, which is known to provide a good description of the SM spectrum avoiding dangerous new sources of flavor violation in the dimension-six operators obtained by integrating out heavy fields at the TeV scale [21, 22].

\subsection{Stability}

Having verified that the postulated hierarchy of scales provides a realistic description of the SM spectrum, we proceed to investigate the stability of the Higgs sector in this more complete setup. Building on the discussion of section 3.2, we carefully consider the impact of the vector-like fermions and the corresponding intergenerational couplings. Once again, our primary concern is to ensure the stability of $m_{3}$, which is the lightest scale of the model.

On general grounds, the largest ratio of mass parameters in the model is $m_{1}^{2} / m_{3}^{2} \sim \varepsilon^{-8}$. Hence the $m_{1}^{2}$ contribution to $\delta m_{3}^{2}$ needs to be suppressed by 4 loop factors (or, equivalently, small couplings), in order to avoid an unreasonably large tuning. A systematic search for diagrams with potentially problematic contributions needs to extend up to the 3 -loop order. What protects the hierarchy of scales is the largely decoupled nature of the fields living on the different sites. Any interaction between them necessarily involve the link fields. The link between $H_{3}$ and $H_{1}$ must therefore be mediated through two link fields and some field living on the second site. Any diagram complicated enough to contain all of these fields necessarily appear at the 3 -loop order or higher, thereby protecting $m_{3}^{2}$ against large corrections. 


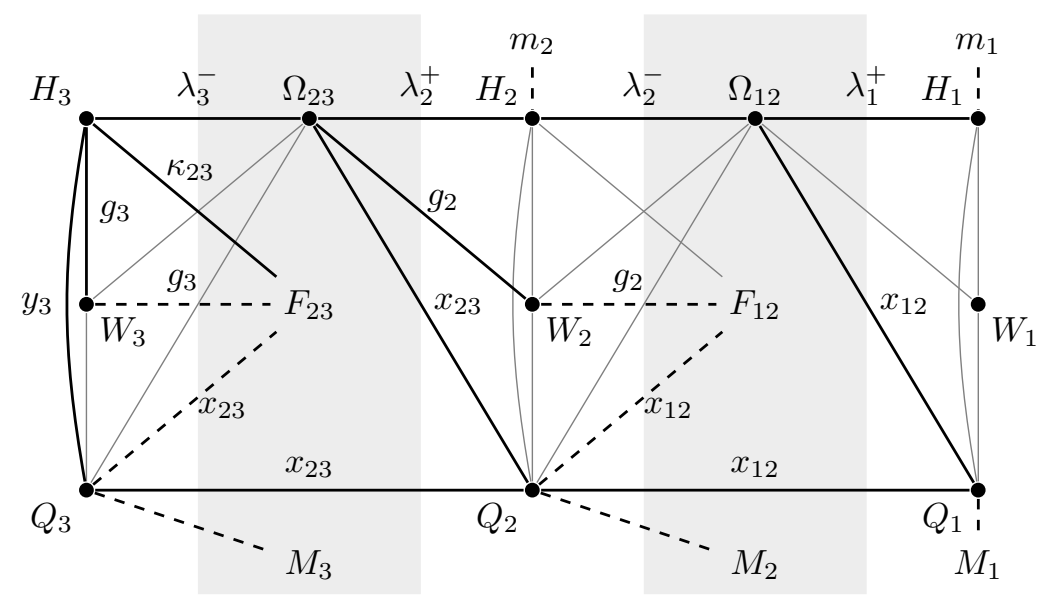

Figure 1. Diagram showing how to connect $H_{3}$ (top left) to the various mass parameters of the theory at a given order in the expansion in terms of loops (in one-to-one correspondence with the lines) and couplings (indicated over each line), within the full model (see main text). The faint gray lines correspond to loops/couplings which are less dangerous for the stability of the hierarchy. The vertical bands groups fields and mass parameters associated to different energy scales.

To make our reasoning more systematic, we consider the known topological relations between the loop order of a diagram and the couplings this diagram contains. A mass correction diagram to $H_{3}$ has two external legs, and the loop order $\ell$ is given by

$$
2 \ell=2 n_{\lambda}+n_{g}+n_{x}+n_{y}+2 n_{\kappa}-n_{F},
$$

where $n_{\lambda}$ is the number of $\lambda_{i}^{ \pm}$couplings; $n_{g}$ denotes the number of gauge couplings $\left(g_{i}\right) ; n_{y}$ and $n_{x}$ indicate the number of Yukawa couplings $y_{i}\left(\hat{y}_{i}\right)$ and $x_{i j}\left(\hat{x}_{i j}\right)$, respectively; $n_{\kappa}$ is the number of trilinear scalar couplings $\left(\kappa_{i j}\right)$; and $n_{F}$ denotes the number of VEV insertions $\left(F_{i j}\right)$. The possible couplings among fields are limited: the ways in which the different fields couple to one another is summarized by the diagram in figure 1 .

The diagram in figure 1 provides an effective tool for a systematic analysis of the stability of $m_{3}$. To do so, we need to begin and end at $H_{3}$ in the top left corner, corresponding to considering a two-point diagram with two external $H_{3}$ lines, and connect to some other mass scale. For instance, we can connect $H_{3}$ to $\Omega_{23}$ via the $\lambda_{3}^{-}$coupling, and from there to the $H_{2}$ field via $\lambda_{2}^{+}$. At this point, we can get an $m_{2}^{2}$ mass-insertion, generating a correction to $m_{3}^{2}$ proportional to $m_{2}^{2}$. This is the shortest way to go from $H_{3}$ to $m_{2}$ : any other path would involve more couplings and, correspondingly, a stronger loop suppression. From this minimal path we deduce that $m_{3}^{2}$ receives a correction from $m_{2}^{2}$ at the 2-loop order, with coefficient $\lambda_{3}^{+} \lambda_{2}^{-}$. A point of caution: the figure does not guarantee the existence of a diagram, but it can easily be checked whether one exists given the couplings involved. In general, $H_{3}$ can be connected to any of the masses and VEVs in the figure, providing the end points of any line, picking up all the couplings along the way. ${ }^{4}$ Then one can trace a path back to $H_{3}$ (typically the same path) to connect to the second $H_{3}$ leg of a mass

\footnotetext{
${ }^{4}$ The quartic couplings should be counted only once in the paths back and forth.
} 
correction diagram. From eq. (4.15), it follows that every one of the solid lines in the figure increases the resulting Feynman diagram by 1-loop order (half going out and half coming back again).

From the inspection of the diagram in figure 1 we identify three potentially problematic contributions to $\delta m_{3}^{2}$, i.e. contributions where the loop suppression alone is not enough to ensure the smallness of the corrections. These correspond to the paths connecting $H_{3}$ to $F_{12}$ or $M_{2}$ at the 2-loop level. The possibility of coupling $H_{3}$ to $W_{2}$ and then $F_{12}$ through $\lambda_{3}^{-}$and $g_{2}$ was already identified in section 3.2: the resulting contribution is

$$
\delta m_{3}^{2} \sim \frac{1}{(4 \pi)^{4}} \lambda_{3}^{-} g_{2}^{4} F_{12}^{2} \sim \frac{\lambda_{3}^{-}}{\varepsilon^{2}} m_{3}^{2}
$$

and can ultimately be tamed by requiring a small $\lambda_{3}^{-}$, cf. condition (3.23). The seemingly problematic contributions induced by the heavy fermions arise from coupling $H_{3}$ to $Q_{2}$. Following the path via $y_{3}\left(\hat{y}_{3}\right)$ and $x_{23}\left(\hat{x}_{23}\right)$, the mass insertions on the $Q_{2}$ line implies

$$
\overbrace{Q_{2}}^{\Omega_{23}}-\bar{H}_{3} \rightarrow \delta m_{3}^{2} \sim \frac{1}{(4 \pi)^{4}} \hat{y}_{3}^{2} x_{23}^{2} M_{2}^{2} \sim \frac{x_{23}^{2}}{\varepsilon^{2}} m_{3}^{2} .
$$

It is easy to realize that this is not a problematic contribution once we assume

$$
\text { II) } x_{i j}=\mathcal{O}(\epsilon) \text {, }
$$

which is required already at the tree level in order to ensure the hierarchy in eq. (4.5). Incidentally, we note that having somewhat suppressed intergenerational couplings is easily reconciled with the $5 \mathrm{D}$ picture, where such couplings arise from overlap integrals. The second 2-loop path from $H_{3}$ to $Q_{2}$ on figure 1 is suppressed by both $\lambda_{3}^{-}$and $x_{23}\left(\hat{x}_{23}\right)$ and therefore sub-dominant.

By inspection of the other paths in figure 1, one quickly realizes that there are no other problematic radiative contributions to $m_{3}$. Furthermore, we note that the next-tolightest mass, $M_{3}$, is protected from $M_{2}$ by the smallness of $x_{23}\left(\hat{x}_{23}\right)$ and by loop factors, ensuring its stability. We thus conclude that if the two conditions in eq. (3.23) and (4.18) are satisfied, the mass hierarchy of the full model is stable under radiative corrections.

\section{Conclusions}

Models addressing the hierarchy problem via flavor-blind dynamics are in strong tension with high- $p_{T}$ experiments. On the other hand, signals of flavor-non-universal interactions seem to emerge from low-energy experiments [12]. Motivated by these observations, we have analyzed the stability of the Higgs sector of a model addressing the flavor problem via a hierarchical spectrum of scalar, fermion, and flavor-non-universal massive gauge fields. The spectrum of new states spans three orders of magnitude: from the TeV scale up to about $10^{3} \mathrm{TeV}$. 
The model we have considered, which is only a toy model, provides a representative example of more realistic constructions based on the idea that the generation index refers to a specific localization in an extra space-like dimension. As we have shown, this construction provides an a posteriori explanation of the observed hierarchies in the SM fermion spectrum in terms of the scale hierarchies in the underlying theory: the light generations are light not because of small couplings, but because their effective coupling to the Higgs field is mediated by the exchange of heavy new states. A similar phenomenon occurs for the smallness of the off-diagonal entries of the CKM matrix.

In such a context, one might be worried about the impact of the heavy states in destabilizing the SM ground state. Our analysis shows that the effective mass term of the SM-like Higgs field does not receive large corrections proportional to the heavy fields. More precisely, we have shown that the Higgs mass receives at most relative corrections of $\mathcal{O}(1)$, provided the two conditions in eqs. (3.23) and (4.18) are satisfied. The condition (4.18) is the natural requirement of a not too-large mixing between SM-like and heavy vector-like fermions. Condition (3.23), which is the only real tuning of the model, is nothing but a manifestation of the little hierarchy problem in this context: an $\mathcal{O}(1 \%)$ tuning that we need to impose in order to screen the electroweak scale from the lightest NP states of the model, which are expected to be at the TeV scale. This latter problem could be addressed in more sophisticated versions of the model, with additional protective symmetries acting on the Higgs sector; however, this is beyond of the scope of this paper.

In summary, our analysis provides a concrete example of how to convert scale hierarchies into flavor hierarchies, in the context of models with flavor-non-universal gauge interactions, and the proof that this exchange does not worsen the electroweak hierarchy problem. Actually, this type of construction makes the electroweak hierarchy problem less severe compared to flavor-blind frameworks, due to the weaker bounds on $\mathrm{TeV}$-scale NP coupled mainly to the third generation.

\section{Acknowledgments}

This project has received funding from the European Research Council (ERC) under the European Union's Horizon 2020 research and innovation program under grant agreement 833280 (FLAY), and by the Swiss National Science Foundation (SNF) under contract 200021-175940. 
Open Access. This article is distributed under the terms of the Creative Commons Attribution License (CC-BY 4.0), which permits any use, distribution and reproduction in any medium, provided the original author(s) and source are credited.

\section{References}

[1] G.F. Giudice, Naturalness after LHC8, PoS EPS-HEP2013 (2013) 163 [arXiv:1307.7879] [INSPIRE].

[2] G. 't Hooft et al. eds., Recent Developments in Gauge Theories, Proceedings, Nato Advanced Study Institute, Cargese, France, August 26-September 8, 1979 [NATO Sci. Ser. B 59 (1980) 1] [INSPIRE].

[3] G. Isidori, Y. Nir and G. Perez, Flavor Physics Constraints for Physics Beyond the Standard Model, Ann. Rev. Nucl. Part. Sci. 60 (2010) 355 [arXiv:1002.0900] [InSPIRE].

[4] R.S. Chivukula and H. Georgi, Composite Technicolor Standard Model, Phys. Lett. B 188 (1987) 99 [INSPIRE].

[5] G. D'Ambrosio, G.F. Giudice, G. Isidori and A. Strumia, Minimal flavor violation: An Effective field theory approach, Nucl. Phys. B 645 (2002) 155 [hep-ph/0207036] [inSPIRE].

[6] D.B. Kaplan, Flavor at SSC energies: A New mechanism for dynamically generated fermion masses, Nucl. Phys. B 365 (1991) 259 [InSPIRE].

[7] T. Gherghetta and A. Pomarol, Bulk fields and supersymmetry in a slice of AdS, Nucl. Phys. B 586 (2000) 141 [hep-ph/0003129] [INSPIRE].

[8] R. Contino, Y. Nomura and A. Pomarol, Higgs as a holographic pseudoGoldstone boson, Nucl. Phys. B 671 (2003) 148 [hep-ph/0306259] [INSPIRE].

[9] K. Agashe, G. Perez and A. Soni, Flavor structure of warped extra dimension models, Phys. Rev. D 71 (2005) 016002 [hep-ph/0408134] [INSPIRE].

[10] G. Panico and A. Pomarol, Flavor hierarchies from dynamical scales, JHEP 07 (2016) 097 [arXiv: 1603.06609] [INSPIRE].

[11] M. Bordone, C. Cornella, J. Fuentes-Martin and G. Isidori, A three-site gauge model for flavor hierarchies and flavor anomalies, Phys. Lett. B 779 (2018) 317 [arXiv:1712.01368] [INSPIRE].

[12] LHCb collaboration, Experimental Review on Lepton Universality and Lepton Flavour Violation tests in B decays, EPJ Web Conf. 234 (2020) 01004 [INSPIRE].

[13] N. Arkani-Hamed, A.G. Cohen and H. Georgi, (De)constructing dimensions, Phys. Rev. Lett. 86 (2001) 4757 [hep-th/0104005] [INSPIRE].

[14] Y. Fujimoto, T. Nagasawa, K. Nishiwaki and M. Sakamoto, Quark mass hierarchy and mixing via geometry of extra dimension with point interactions, PTEP 2013 (2013) 023B07 [arXiv: 1209.5150] [INSPIRE].

[15] C.T. Hill, S. Pokorski and J. Wang, Gauge Invariant Effective Lagrangian for Kaluza-Klein Modes, Phys. Rev. D 64 (2001) 105005 [hep-th/0104035] [INSPIRE].

[16] H.-C. Cheng, C.T. Hill, S. Pokorski and J. Wang, The Standard Model in the Latticized Bulk, Phys. Rev. D 64 (2001) 065007 [hep-th/0104179] [INSPIRE]. 
[17] R.A. Porto and A. Zee, The Private Higgs, Phys. Lett. B 666 (2008) 491 [arXiv:0712.0448] [INSPIRE].

[18] G.F. Giudice and O. Lebedev, Higgs-dependent Yukawa couplings, Phys. Lett. B 665 (2008) 79 [arXiv: 0804.1753] [INSPIRE].

[19] C.T. Hill, P.A.N. Machado, A.E. Thomsen and J. Turner, Scalar Democracy, Phys. Rev. D 100 (2019) 015015 [arXiv: 1902.07214] [INSPIRE].

[20] Particle Data Group collaboration, Review of Particle Physics, PTEP 2020 (2020) 083C01 [INSPIRE].

[21] R. Barbieri, G. Isidori, J. Jones-Perez, P. Lodone and D.M. Straub, U(2) and Minimal Flavour Violation in Supersymmetry, Eur. Phys. J. C 71 (2011) 1725 [arXiv:1105.2296] [INSPIRE].

[22] R. Barbieri, D. Buttazzo, F. Sala and D.M. Straub, Flavour physics from an approximate $\mathrm{U}(2)^{3}$ symmetry, JHEP 07 (2012) 181 [arXiv:1203.4218] [INSPIRE]. 